\title{
Epigenetic clock at birth and psychiatric problems during early childhood
}

\author{
Suarez, Anna
}

2017-09

Suarez , A , Lahti , J , Czamara , D , Lahti , M , Knight , A K, Girchenko , P , Hämäläinen , E , Kajantie , E , Laivuori , H, Villa , P M , Reynolds , R M , Smith , A K, Binder , E B \& Räikkönen , K 2017 , ' Epigenetic clock at birth and psychiatric problems during early childhood ' , Psychoneuroendocrinology , vol. 83S , pp. 52-52 . https://doi.org/10.1016/j.psyneuen.2017.07.381

http://hdl.handle.net/10138/297941

https://doi.org/10.1016/j.psyneuen.2017.07.381

publishedVersion

Downloaded from Helda, University of Helsinki institutional repository.

This is an electronic reprint of the original article.

This reprint may differ from the original in pagination and typographic detail.

Please cite the original version. 


\section{Life stress and epigenetic aging}

Anthony Zannas

Max Planck Institute of Psychiatry, Germany

E-mail address: aszannas@gmail.com.

Background: Stress-related phenotypes are associated with accelerated aging and increased risk for aging-related diseases, but the underlying molecular mechanisms are poorly understood.

Methods: This session will examine the impact of different types of stress on epigenetic aging, a composite measure derived from several DNA methylation sites that has been associated with increased risk for aging-related disease phenotypes.

Results: Life stressors accelerate epigenetic aging. In particular, accelerated epigenetic aging is associated with cumulative life stress, personal stressors, and certain types of early life adversity, whereas complex interactions exist among advancing age, early life stress, and cumulative stress exposure. Notably, the impact of early life stress on epigenetic aging may be prevented by certain preventive strategies. At the molecular level, the effects of stress on epigenetic aging may be mediated by stress-induced aberrant glucocorticoid signaling, as suggested by the co-localization of epigenetic clock methylation sites with glucocorticoid response elements and the functional changes observed at these sites upon glucocorticoid exposure. The glucocorticoid-responsive epigenetic clock sites show enriched association for aging-related diseases, including coronary artery disease, arteriosclerosis, and leukemias.

Conclusions: Both cumulative life stress and certain types of early life trauma can accelerate epigenetic aging, effects that may be driven by glucocorticoid-induced epigenetic changes and may be prevented by interventions targeted in early life. These findings contribute to our understanding of mechanisms linking lifetime stress with heightened disease risk.

http://dx.doi.org/10.1016/j.psyneuen.2017.07.379

\section{Aerobic training increases telomere length in family caregivers: A randomized trial}

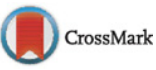

Eli Puterman

University of British Columbia, Canada

E-mail address: eli.puterman@ubc.ca.

Background: Habitual physical activity is associated with longer telomeres in immune cells. Previous observational studies have shown that an active lifestyle especially benefits individuals experiencing high levels of life stress. To date, it remains unexplored whether the mitigating effects of physical activity on telomere shortening in high stressed adults translate to inactive individuals who become active.

Methods: 68 female $(N=55)$ and male $(N=13)$ previously inactive, high stressed $(\geq 0.5$ SD above national norms on Perceived Stress Scale) were equally randomized ( $N=34 /$ group) to either a 6 -month aerobic training program or a waitlist control group. All participants underwent a fasting blood draw at baseline and at trial's end. Leukocyte telomere length (TL) was measured with quantitate polymerase chain reaction $t / s$ ratio and converted to base pairs for interpretation. Covariates in models included age, body mass index, and sex.

Results: 64 participants completed the 6 month intervention $(N=31$ in aerobic arm; $N=33$ in waitlist control arm). Intent-totreat mixed effect model with maximum likelihood estimation demonstrated a significant treatment effect [treatment* time beta $=70.75(\mathrm{SE}=33.98), p=.041]$. Within group analyses demon- strated a significant 65.49 base pairs increase in TL in the aerobic arm and a non-significant 5.25 base pairs decrease in the waitlist arm. Results from sensitivity analyses with dropout's baseline TL data carried forward were similar.

Conclusion: This study is the first exercise-based randomized trial to demonstrate telomere lengthening. This study offers promise that cellular aging can potentially be reversed in previously inactive and highly stressed adults.

\section{http://dx.doi.org/10.1016/j.psyneuen.2017.07.380}

\section{Epigenetic clock at birth and psychiatric problems during early childhood}

Anna Suarez ${ }^{1, *}$, Jari Lahti ${ }^{1}$, Darina Czamara ${ }^{2}$, Marius Lahti ${ }^{1}$, Anna K. Knight ${ }^{3}$, Polina Girchenko ${ }^{1}$, Esa Hämäläinen ${ }^{4,5}$, Eero Kajantie $^{5,6,7}$, Hannele Laivuori ${ }^{1,5,8}$, Pia M. Villa ${ }^{1,5}$, Rebecca M. Reynolds ${ }^{9}$, Alicia K. Smith ${ }^{10}$, Elisabeth B. Binder ${ }^{2,10}$, Katri Räikkönen ${ }^{1}$

${ }^{1}$ University of Helsinki, Finland

${ }^{2}$ Max-Planck Institute of Psychiatry, Germany

${ }^{3}$ Genetics and Molecular Biology Program, Emory

University, USA

${ }^{4}$ HUSLAB, Finland

${ }^{5}$ Helsinki University Central Hospital, Finland

${ }^{6}$ Oulu University Hospital and University of Oulu, Finland

${ }^{7}$ National Institute for Health and Welfare, Finland

${ }^{8}$ Institute for Molecular Medicine, Finland

${ }^{9}$ British Heart Foundation Centre for Cardiovascular

Science, Queen's Medical Research Institute,

University of Edinburgh, UK

${ }^{10}$ Emory University School of Medicine, USA

E-mail address: anna.suarez@helsinki.fi (A. Suarez).

Background: A novel biomarker of aging at birth, namely epigenetic gestational age (GA) based on DNA methylation in fetal cord blood, has been recently developed to accurately estimate GA at birth. We examined whether this aging biomarker prospectively predicted psychiatric problems during early childhood.

Methods: 407 mothers from the PREDO Study filled in the Child Behavior Checklist at child's mean age of $3.7(\mathrm{SD}=0.75)$ years. DNA was extracted from cord blood and methylation was analyzed with Illumina 450k array. DNA methylation (DNAm) GA of fetal cord blood DNA was calculated from $148 \mathrm{CpG}$ sites shown to have high accuracy in predicting chronological GA. Epigenetic GA was calculated as arithmetic difference between DNAm GA and chronological GA.

Results: In boys only child lower epigenetic GA (lower DNAm GA compared to chronological GA) was prospectively associated with higher total behavioral $(p=0.047)$, emotionally reactive $(p=0.035)$, and withdrawn $(p=0.004)$ problems in models adjusting for chronological GA, cord blood cell-type composition, genetic population stratification, and child age at the time of testing.

Conclusions: Epigenetic GA immaturity seems to be developmentally disadvantageous for boys, who in early childhood show higher psychiatric problems in maternal reports.

http://dx.doi.org/10.1016/j.psyneuen.2017.07.381 